\title{
Synthesis of High Polymers with a Light Absorption Band in the Visible Region by Interfacial Polycondensation Reaction
}

\author{
Motoharu Shiba, Hidenori Hiramasu, Hiroto NaKano, \\ and Yasuaki Kawano \\ Research Laboratory, Chugai Pharmaceutical Co., Ltd., \\ Takada, Toshima, Tokyo, Japan. \\ Yoshimichi SHIGERI and Tamotsu Kondo \\ Faculty of Pharmaceutical Sciences, Science University of Tokyo, \\ Funagawara, Shinjuku, Tokyo, Japan.
}

(Received June 24, 1972)

\begin{abstract}
Several commercially available dyes having two or more chemically active hydrogen atoms in their molecules were allowed to react with sebacoyl chloride to form polymers by interfacial polycondensation. Infrared spectra of the products showed absorption bands indicating the formation of polymers. The polymers were found to absorb visible light in solution. The position of $\lambda_{\max }$ of their visible spectra was the same as that of the visible spectra of monomer dyes. The shift of $\lambda_{\max }$ was observed when the solvent was changed.

KEY WORDS Interfacial Polycondensation / Commercial Dyes / Absorption of Visible Light / Sebacoyl Chloride /
\end{abstract}

In the course of previous works on microcapsules, ${ }^{1-5}$ it has been found that the process of interfacial polycondensation makes it possible to synthesize many new high polymers under mild conditions, which otherwise could not be obtained. Thus, for example, poly(dimethylaminostilbene phthalamide $)^{4}$, a fluorescent polymer or poly(L-lysine phthalamide $)^{5}$, a protein-like polymer was prepared at low temperatures and atmospheric pressure by the interfacial polycondensation reaction of diaminostilbene or Llysine in water with $p$-phthaloyl dichloride in a chloroform-cyclohexane mixture.

This has prompted the authors to make an attempt to synthesize polymers with a light absorption band in the visible region by the interfacial polycondensation. Such colored polymers, if prepared, would find many applications in the polymer industry as well as in basic research of polymer science. The present paper describes the synthesis of colored polymers by the interfacial polycondensation reaction of commercially available dyes having two or more amino or hydroxyl groups in their molecules in aqueous solution and with sebacoyl chloride in organic solution.

\section{EXPERIMENTAL}

\section{Materials}

The water soluble dyes used in this work were Direct Yellow-4, Direct Orange-10, Direct Red28, Direct Brown-1A, Direct Green-6, and Acid Blue-43. These dyes are commercially available and have chemical structures as shown below. Sebacoyl chloride was a product of Eastman Organic Chemicals, U.S.A. All other chemicals used were of reagent grade.

\section{Polycondensation}

The polycondensation was carried out in a flask containing $500 \mathrm{ml}$ of water, $0.005 \mathrm{~mol}$ of a dye, and $0.01 \mathrm{~mol}$ of sodium hydroxide. To this solution was added a solution of $0.005 \mathrm{~mol}$ of sebacoyl chloride in $100 \mathrm{ml}$ of chloroform at one time with vigorous stirring at room temperature, and the stirring was further continued for $30 \mathrm{~min}$. At the end of this period, the dispersion was centrifuged after adding $100 \mathrm{~m} l$ of cyclohexane to it. The polymer was then ob- 


$$
\text { (n) }
$$

tained as a precipitate. The polymer was dried in a vacuum oven at temperatures below $100^{\circ} \mathrm{C}$, and the dried polymer dispersed in distilled water. The polymer dispersion was dialysed in cellophane tubing against sodium hydroxide solution at room temperature until the outer solution became colorless. Finally, an optimum volume of aqueous calcium chloride solution was added to the polymer dispersion in the cellophane tubing. The precipitate was centrifuged and dried at temperature below $100^{\circ} \mathrm{C}$.

Measurement of Absorption Spectra and Viscosity

Infrared spectra were recorded on a Hitachi 225 Type infrared spectrometer using potassium bromide pellets. Visible and ultraviolet absorption spectra were obtained by a Hitachi EPS-2 Type spectrometer. Inherent viscosities $\left(\eta_{\mathrm{inh}}=\right.$ $\left.\left[\ln \eta_{\mathrm{re} 1}\right] / c\right)$ of all polymers synthesized were determined at $30^{\circ} \mathrm{C}$ in dimethylformamide by an Ostwald viscometer, with solution of a concentration of $0.5 \mathrm{~g} / 100 \mathrm{~m} l$. In some cases, reduced viscosities $\left(\eta_{\mathrm{sp}} / c\right)$ were also determined at $30^{\circ} \mathrm{C}$ at several concentrations in distilled water or aqueous sodium hydroxide solutions using an Ubbelohde viscometer.

\section{RESULTS AND DISCUSSION}

Table I summarizes the data on the yield, inherent viscosity, and color of the polymers prepared. The polymers were visually indistinguishable from each other because of their dark color while the monomer dyes had distinctive colors. The inherent viscosities varied with monomer dye to some extent. The polymer derived from DB-1A had a moderate inherent viscosity $\left(\eta_{\mathrm{inh}}, 0.3\right)$, indicating this to have the highest molecular weight among the poly- 
Table I. Yield and inherent viscosity for several polydyes

\begin{tabular}{lccl}
\hline & Yield, $\%$ & $\eta_{\text {inh }}$ & \multicolumn{1}{c}{ Color } \\
\hline PDY-4 & 10.1 & 0.163 & very dark yellow \\
PDO-10 & 11.3 & 0.211 & dark red \\
PDR-28 & 13.7 & 0.178 & very dark red \\
PDB-1A & 21.0 & 0.314 & very dark brown \\
PDG-6 & 14.2 & 0.208 & dark green \\
PAB-43 & 16.8 & 0.263 & blue black
\end{tabular}

mers prepared in this work. On the other hand, low-molecular-weight polymers $\left(\eta_{\mathrm{inh}}, 0.2\right.$ or below) were obtained from other dyes. Since the optimum condition for the polycondensation reaction involving dyes should depend on the molecular structure of monomer dye used, the viscosity value in Table I would not necessarily be a proper measure of the reactivity of dye.

In Table II are shown the solubilities in various organic solvents of polymers and monomer dyes. In general, monomer dyes were soluble in solvents that were good solvents for the polymers derived from them. Dimethylformamide was the best of the solvents tested in this work for all polymers. With the exception

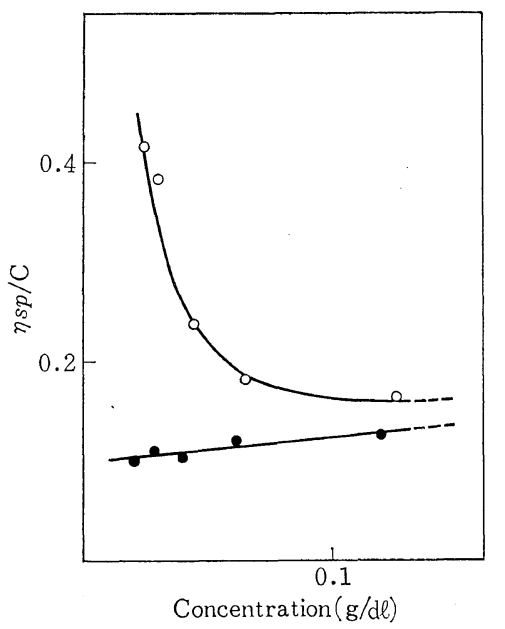

Figure 1. Reduced viscosity of PDR-28: - $\mathrm{O}-$, distilled water; - $-0.005-N$ aqueous $\mathrm{NaOH}$.

of PDB-1A and PAB-43, all the other polymers were soluble in aqueous sodium hydroxide solution. The addition of EDTA-2Na to the aqueous dispersion of these two polymers caused the dissolution of polymers probably owing to the removal of calcium ions by the chelating agent.

In Figure 1 are shown the reduced viscosities

Table II. Solubility of polymers and monomers in various solvents ${ }^{\mathrm{a}}$

\begin{tabular}{|c|c|c|c|c|c|c|c|c|c|c|c|c|}
\hline & \multicolumn{2}{|c|}{ DY -4} & \multicolumn{2}{|c|}{ DO-10 } & \multicolumn{2}{|c|}{ DR-28 } & \multicolumn{2}{|c|}{ DB-1A } & \multicolumn{2}{|c|}{ DG-6 } & \multicolumn{2}{|c|}{$\mathrm{AB}-43$} \\
\hline & $\mathbf{P}$ & $\mathbf{M}$ & $\mathbf{P}$ & $\mathbf{M}$ & $\mathbf{P}$ & $\mathbf{M}$ & $\mathbf{P}$ & $\mathbf{M}$ & $\mathbf{P}$ & $\mathbf{M}$ & $\mathbf{P}$ & $\mathbf{M}$ \\
\hline Ethyl alcohol & $\triangle$ & & $\triangle$ & & $x$ & & $\times$ & & $x$ & & $x$ & \\
\hline Benzyl alcohol & $\bigcirc$ & $\triangle$ & $\triangle$ & & $\triangle$ & & $\bigcirc$ & & 0 & & $\triangle$ & \\
\hline Acetone & $\times$ & $\triangle$ & $\times$ & $\triangle$ & $x$ & $\triangle$ & $\times$ & $\triangle$ & $\triangle$ & $\triangle$ & $\times$ & \\
\hline Dioxane & $\times$ & $\triangle$ & $x$ & $\triangle$ & $x$ & $\triangle$ & $x$ & $\triangle$ & $x$ & $x$ & $x$ & $\triangle$ \\
\hline Cyclohexanone & $\triangle$ & $\triangle$ & $x$ & $\triangle$ & $\times$ & $\triangle$ & $\times$ & $\triangle$ & 0 & $\times$ & $\times$ & $\triangle$ \\
\hline Dimethylformamide & (a) & $\bigcirc$ & 0 & & (0) & $\bigcirc$ & (0) & $\bigcirc$ & (a) & 0 & 0 & \\
\hline$m$-Cresol & $\times$ & $\triangle$ & $\triangle$ & $\times$ & $x$ & $\times$ & $\times$ & $\times$ & $\triangle$ & $\times$ & $x$ & \\
\hline Methyl ethyl ketone & $\times$ & $\triangle$ & $x$ & $\bigcirc$ & $x$ & $\triangle$ & $x$ & $\triangle$ & $\triangle$ & $\triangle$ & $x$ & $\triangle$ \\
\hline Chloroform & $\times$ & $\times$ & $\triangle$ & $\triangle$ & $\times$ & $x$ & $\times$ & $x$ & $\times$ & $\times$ & $\times$ & $\triangle$ \\
\hline Methylene chloride & $\times$ & $\times$ & $\triangle$ & $\times$ & $x$ & $\times$ & $x$ & $x$ & $\triangle$ & $\times$ & $x$ & $x$ \\
\hline Ethyl acetate & $x$ & $x$ & $\times$ & $x$ & $x$ & $x$ & $x$ & $x$ & $\triangle$ & $x$ & $x$ & $x$ \\
\hline Formic acid & $\times$ & $\times$ & (a) & & $\bigcirc$ & & $\bigcirc$ & & $\triangle$ & & 0 & \\
\hline Tetrahydrofuran & $\times$ & $\triangle$ & $\triangle$ & $\triangle$ & $x$ & $\times$ & $\triangle$ & $\Delta$ & $\bigcirc$ & $\triangle$ & $\times$ & $\triangle$ \\
\hline Cyclohexane & $\times$ & $x$ & $x$ & $x$ & $x$ & $x$ & $x$ & $x$ & $x$ & $\times$ & $\times$ & $x$ \\
\hline Xylene & $\times$ & $\times$ & $\times$ & $x$ & $\times$ & $x$ & $\times$ & $x$ & $\times$ & $\times$ & $\times$ & $x$ \\
\hline Dist water & $\triangle$ & $\bigcirc$ & $\triangle$ & $\bigcirc$ & $\times$ & 0 & $\times$ & & $\times$ & 0 & $\times$ & \\
\hline $0.1-N \mathrm{NaOH}$ solution & 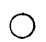 & & & & $\bigcirc$ & & $\triangle$ & & (0) & & $\times$ & \\
\hline
\end{tabular}

a For polymers: (O), highly soluble; $\bigcirc$, moderately soluble; $\triangle$, slightly soluble; $\times$, insoluble. For monomers: $\bigcirc$, soluble; $\triangle$, slightly soluble; $\times$, insoluble. 
of the sodium salt of PDR-28 at several concentrations in distilled water and aqueous $0.005-N$ sodium hydroxide. The figure indicates the same viscostiy behabior for this polymer as for the polyelectrolytes reported in the literature. ${ }^{6}$

The infrared absorption spectra of polymers had characteristic amide I, II, and III bands in region of $1660-1640$ and $1550-1520 \mathrm{~cm}^{-1}$, and around $1250 \mathrm{~cm}^{-1}$, respectively, with the exception of PDY-4. Typical examples of the spectra are

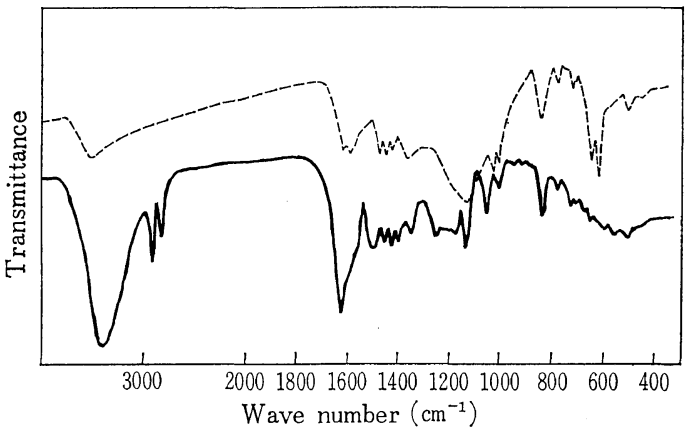

Figure 2. Infrared spectra of DB-1A: mer; ----, monomer.

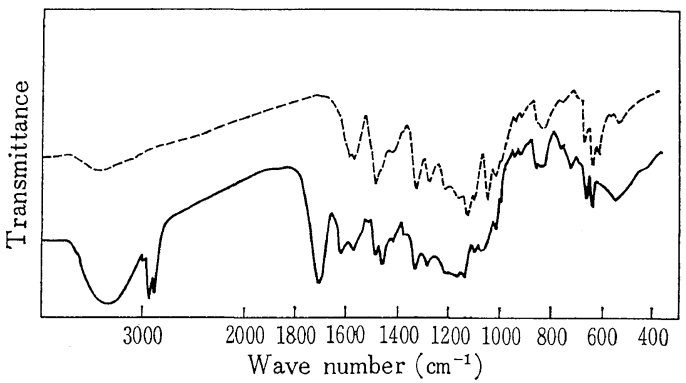

Figure 3. Infrared spectra of DG-6: _ mer; ----, monomer.

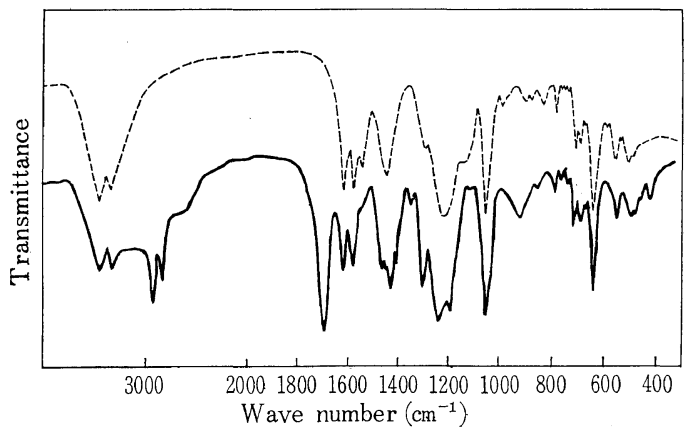

Figure 4. Infrared spectra of AB-43: —, polymer; ----, monomer. given in Figures 2-4. In addition to the above absorption bands, the spectra indicated two absorption bands at 2910 and $2860 \mathrm{~cm}^{-1}$, which were assigned to the $\mathrm{CH}$ vibrations. Moreover, PDY-4, PDG-6, and PAB-43 had a carbonyl streching band at $1700 \mathrm{~cm}^{-1}$ in their spectra due to the ester group. The fact that the amide bands were present while the band due to the ester group is lacking in the spectra of PDB-1A would suggest that hydroxyl groups at the end of their molecules remain unreacted. In the spectra of PDG-6 and PAB-43, however, the amide and ester.bands are clearly seen, suggesting that amino and hydroxyl groups of molecules of the dyes have reacted with sebacoyl chloride.

Infrared spectroscopy alone is not sufficient to establish the presence of azo groups in dye molecules. The azo bands, however, do show up well in the ultraviolet region. ${ }^{7}$ Thus, typical examples of the ultraviolet absorption spectra of polymers in a mixture of methylene chloride and dimethylformamide are given in Figure 5. The spectra in the figure show an azo peak between 360 and $380 \mathrm{~nm}$. The shift in azo absorption might be attributed to the influence of dissociation groups.

Figure 6 gives the visible absorption spectrum

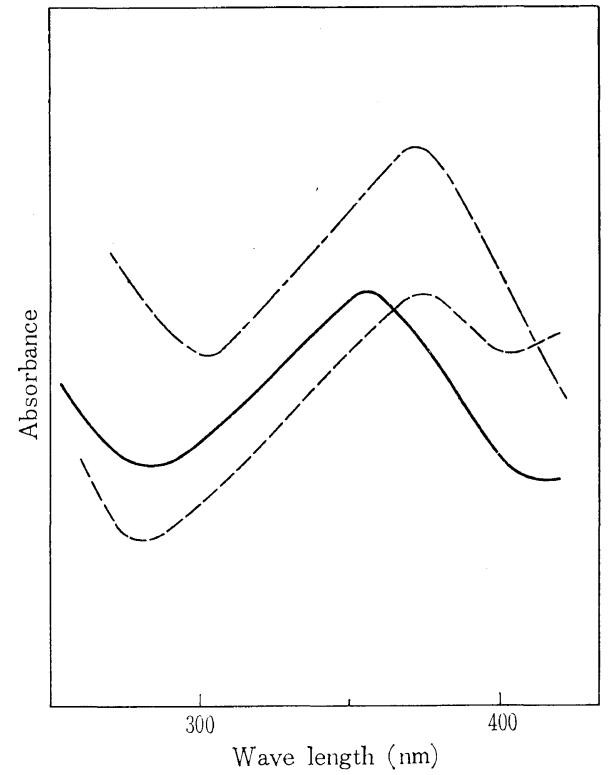

Figure 5. Ultraviolet spectra of polymer: PDR-28; ---, PDB-1A; -.-, PDG-6. 


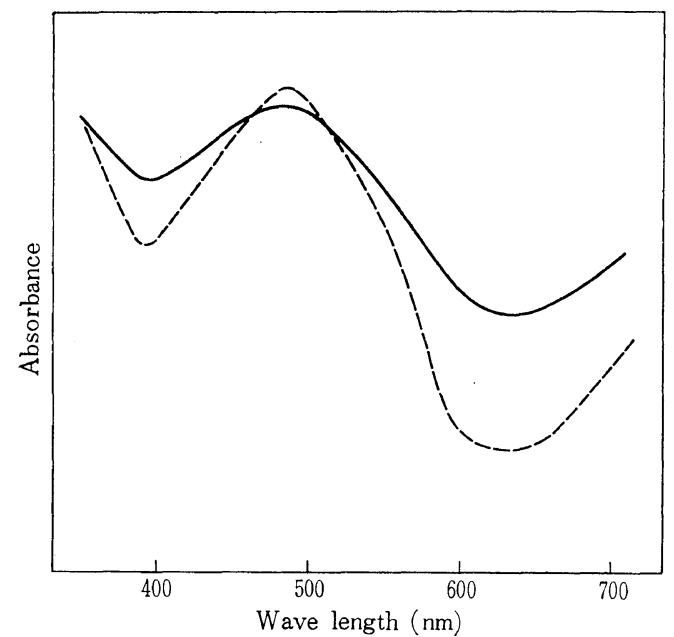

Figure 6. Visible spectra of DR-28 in 0.1-\% aqueous $\mathrm{NaOH}$ : —, polymer; ---, monomer.

of the PDR-28 solution $(0.1-\% \mathrm{w} / \mathrm{v})$ in $0.1-N$ sodium hydroxide, together with that of the DR28 in the same medium. Light absorption changed at all wavelengths to make the shape of the spectrum smooth as compared with that for the monomer dye while the position of $\lambda_{\max }$ remained unchanged. There are two possible reasons for this situation. On the one hand, it might result from the fact that the polymerization dose not produce any considerable modifications in the chromophores of dye molecules though the auxochromes undergo serious chemical changes during the polymerization. On the other hand, it might also be caused by the adsorption of monomer dye molecules on to the polymers in which the chromophores of dye molecules themselves have been subjected to a great alteration through polycondensation.

In order to decide which is more likely the following experiment was carried out. Under the same polymerization conditions as for sebacoyl chloride, benzoyl chloride was allowed to react with the dye in the water phase. The dispersion containing the product was centrifuged and the precipitate dried at a temperature below $100^{\circ} \mathrm{C}$. DB-1A was used because this dye was the most reactive with benzoyl chloride. The product is abbreviated as D-B. Figure 7 shows visible absorption spectra of PDB-1A, DB-1A, and D-B. In dimenthylform-

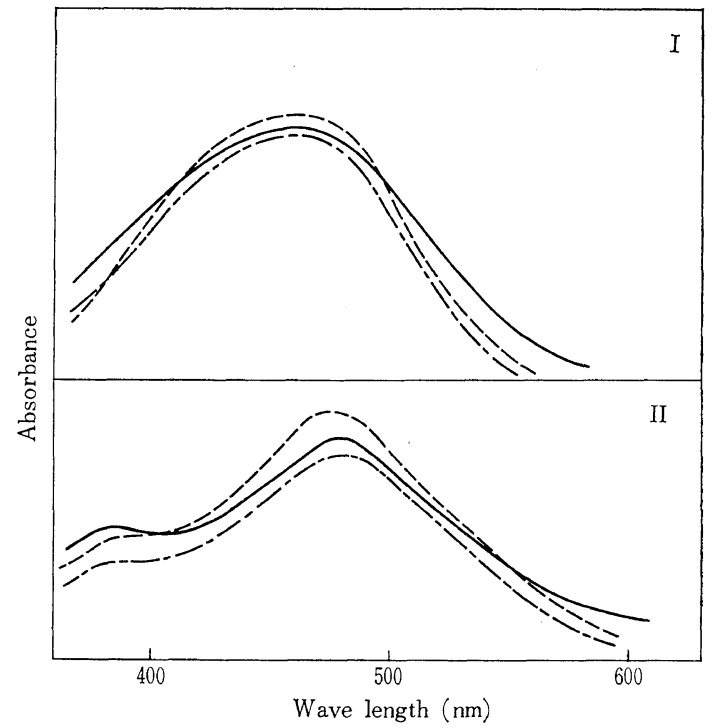

Figure 7. Effect of solvent and auxochrome on visible spectra of DB-1A: I, in dimethylformamide; $\mathrm{II}$, in formic acid; —_, PDB-1A; ---, DB-1A; -.- D-B.

amide $\lambda_{\max }$ was the same for all the three while the absorption peaks of PDB-1A and D-B shifted towards longer wavelengths as compared with DB-1A in formic acid, being indicative of a solvent effect.

Moreover, the absorption spectra of PDY-4 were measured in various solvents to examine the solvent effect on visible absorption spectra. The position of $\lambda_{\max }$ remained unchanged for PDY -4 and DY-4 in cyclohexane, aqueous sodium hydroxide solution, and water, whereas that for the polymer shifted towards a shorter wavelength in dimethylformamide, benzyl alcohol and ethyl alcohol. The polymer was soluble enough in dimethylformamide and aqueous sodium hydroxide to prepare the samples of desired concentrations for the measurements of the absorption spectra while only the saturated solution could be used in the measurements for other solvents. This seems to support the idea that the polymerization causes only slight modification of the chromophores.

\section{REFERENCES}

1. T. M. S. Chang, F. C. MacIntosh, and S. G. Mason, Can. J. Physiol. Pharmacol., 44, 115 (1966). 


\section{Synthesis of Colored High Polymers}

2. S. Suzuki, T. Kondo, and S. G. Mason, Chem. 5. Y. Shigeri, M. Tomizawa, K. Takahashi, M. Pharm. Bull. (Tokyo), 16, 1629 (1968).

Koishi, and T. Kondo, ibid., 49, 3623 (1971).

3. M. Koishi, N. Fukuhara, and T. Kondo, ibid., 6. R. M. Fuoss and U. P. Straus, J. Polym. Sci., 17, 804 (1969). 3, 602 (1948).

4. M. Koishi, N. Fukuhara, and T. Kondo, Can. 7. P. H. Gore and O. H. Wheeter, J. Org. Chem., J. Chem., 47, 3447 (1969). 26, 3295 (1960). 